\title{
Nonlinear Finite Element Analysis of Inflatable Prefolded Membrane Structures under Hydrostatic Loading
}

\author{
M. Haßler, K. Schweizerhof \\ Universität Karlsruhe, Institut für Mechanik
}

2007

Institut für Mechanik

Kaiserstr. 12, Geb. 20.30

76128 Karlsruhe

Tel.: +49 (0) 721/ 608-2071

Fax: +49 (0) 721/ 608-7990

E-Mail: ifm@uni-karlsruhe.de

www.ifm.uni-karlsruhe.de 


\title{
Nonlinear Finite Element Analysis of Inflatable Prefolded Membrane Structures under Hydrostatic Loading
}

\author{
M. Haßler and K. Schweizerhof
}

20. August 2007

Due to their flexibility shell or membrane like structures subjected to gas or fluid loading or gas/fluid support undergo large deformations. In order to describe this deformation dependent loading, where value and direction of the pressure loading are a function of the current configuration of the shell structure, the gas or fluid volumes, which are enclosed by the thin walled structure, have to be considered for the appropriate constitutive equations. Then the numerical formulation of the fluid or gas loading can be derived via an analytical meshfree description for the fluid/gas, which yields a special structure of equations involving the change of the gas or fluid volume respectively the change of the wetted part of the shell surface, see $[2],[11],[12],[13]$. This procedure finally leads to the so-called load-stiffness matrix, to which (in the case of enclosed gas/fluid volumes) several rank updates describing the coupling of the fluid or gas with the structural displacements in addition to the deformation dependence of the pressure load [15] are added. The numerical examples of e.g. a) deploying simply folded membrane structures and b) multi-chamber structures containing fluid and air in arbitrary combination demonstrate, how the simulation of structures with static gas and fluid loading or support can be efficiently performed without discretizing the fluid resp. the gas.

Key words: fluid-structure interaction; hydrostatic loading; gas loading; large displacements; volume dependence; finite elements

\section{Introduction}

Inflatable structures nowadays find a wide field of applications, such as gas supported beams [6] in the field of civil engineering or deployable space structures [4] in aerospace engineering. Even in the field of hydraulic engineering inflatable membranes are used for gas and/or heavy fluid supported dams [5], which allow a direct control of the water retention level just by controlling the internal gas pressure. In conventional simulations these inner state variables (e.g. the internal gas pressure) of gas/fluid supported structures are transferred into pure loading quantities, which are not affected by the deformation. The fact that the applied gas or fluid pressure is volume dependent is often neglected as also for example the volume dependence of the current fluid level.

The aim of this contribution is a large deformation analysis, which includes both the volume and the shape dependence of the inner state variables. For this purpose we refer to former contributions of the authors group [11], [12], [13] and the 
works of Bonet et al. [2], which dealt with gas and fluid supported shell structures and considered both the shape and volume dependence of the hydrostatic pressure distribution. With these derivations it is possible to come up with an analytical formulation of the fluid/gas only described by surface integrals over the surrounding wetted structure. Hence no discretization of the fluid or the gas e.g. by FE or boundary elements is necessary. A further contribution of the authors group currently being in progress [7] merges all these results and thus provides a unified formulation for closed chambers containing any combination of volume dependent loading. Therefore the notations in the current contribution were adopted from [7].

First the necessary governing equations of the problem will be briefly derived via their virtual work expressions. In a further step the obtained variation of the gas/fluid potential has to be linearized for a Newton-type solution algorithm. After FEdicretization of the linearized weak form the so-called load stiffness matrix (see also [14], [15]) is obtained, which is updated by several dyadic products, reflecting the volume dependence of the inner state variables. The numerical examples contain large deformation analyses of fluid and gas filled multi chamber systems with rather thin flexible walls and illustrate the performance of the derived algorithm.

\section{Governing Equations}

The general problem of thin walled shells containing gas and/or fluid (see figure 2.1) is prescribed by its properties of the potential energy $\Pi$, which consists of the - here for simplicity - elastic potential $\Pi^{e l}$ of the surrounding structure of the containment, the potential $\Pi^{f s i}$ of the internal fluid and gas and the work $W^{e x}$ of the external forces. For equilibrium the variation of the total potential energy then vanishes identically.

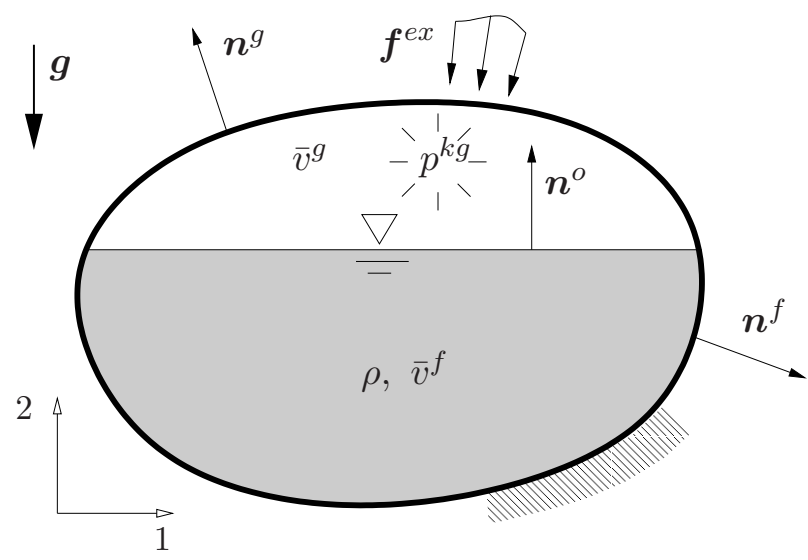

Bild 2.1: Elastic shell structure filled with fluid and gas

\subsection{Virtual Work Expression}

The variation $\delta \Pi$ of the total potential energy is given by the variation of its parts:

$$
\delta \Pi=\delta \Pi^{e l}+\delta \Pi^{f s i}-\delta W^{e x}=0
$$

For the elastic energy and the work of the standard external loading, such as e.g. dead loading, we refer to standard FE-text books [17], [19]. In the following the focus 
is only on the potential of the fluid-structure-interaction $(f s i)$ part and the gas or fluid loading,

$$
\delta \Pi^{f s i}=\delta V^{f}-\delta W^{g}-\delta W^{f},
$$

which consists of the virtual gravity potential $\delta V^{f}$ of the heavy fluid, the virtual work $\delta W^{g}$ of the gas compression and the virtual work $\delta W^{f}$ of the fluid compression, which is actually zero for an incompressible fluid. Because all terms have already been derived in detail in [7] and [10], they will only be briefly presented in this contribution. The virtual potential

$$
\delta V^{f}=-\delta \rho \boldsymbol{g} \cdot \overline{\boldsymbol{s}}^{f}-\rho \boldsymbol{g} \cdot \delta \overline{\boldsymbol{s}}^{f}
$$

due to acceleration of gravity $\boldsymbol{g}$ can be written in terms of the fluid density $\rho$ and its first order volume moment

$$
\bar{s}=\int_{\bar{v}^{f}} \boldsymbol{x}^{f} d v .
$$

The virtual work of the gas volume compression (see also [18])

$$
\delta W^{g}=p^{k g} \delta \bar{v}^{g}
$$

consists of the gas volume change $\delta \bar{v}^{g}$ and of the volume dependent gas pressure

$$
p^{k g}\left(\bar{v}^{g}\right)^{\kappa}=\text { const } \quad(\text { with the isentropic exponent } \kappa) .
$$

\subsection{Boundary Integral Representation of the Geometry}

In section 2.1 the variation of the fluid potential was given in terms of the variables $\delta \bar{v}^{g}$ and $\delta \overline{\boldsymbol{s}}^{f}$ describing the geometrical attributes of the fluid and gas. Consequently the second step will now be the representation of these geometrical variables by boundary integrals, enabling an analytical meshfree description of the enclosed gas and heavy fluid via their surrounding wetted surface. Sectioning both control volumes $\bar{v}^{g}$ and $\bar{v}^{f}$ in figure 2.1 yields along with a three dimensional projection of the geometry the necessary virtual geometrical variables. Thus we obtain the virtual gas volume and the virtual first order volume moment of the heavy fluid as:

$$
\begin{aligned}
& \delta \bar{v}^{g}=\int_{\eta^{g}} \int_{\xi^{g}} \boldsymbol{n}^{g} \cdot \delta \boldsymbol{u}^{g} d \xi d \eta-\int_{\eta^{o}} \int_{\xi^{o}} \boldsymbol{n}^{o} \cdot \delta \boldsymbol{u}^{o} d \xi d \eta, \\
& \delta \overline{\boldsymbol{s}}^{f}=\int_{\eta^{f}} \int_{\xi^{f}}\left(\boldsymbol{n}^{f} \cdot \delta \boldsymbol{u}^{f}\right) \boldsymbol{x}^{f} d \xi d \eta+\int_{\eta^{o}} \int_{\xi^{o}}\left(\boldsymbol{n}^{o} \cdot \delta \boldsymbol{u}^{o}\right) \boldsymbol{x}^{o} d \xi d \eta .
\end{aligned}
$$

In equations (2.7) and (2.8) $\boldsymbol{n}^{g}$ denotes the non-normalized normal vector on the gas boundary and $\boldsymbol{n}^{f}$ and $\boldsymbol{n}^{o}$ the non-normalized normal vectors on the fluid boundary and on the fluid level. $\boldsymbol{x}^{f}$ and $\boldsymbol{x}^{o}$ define the position vectors to a wetted structural point and to the fluid surface. $\delta \boldsymbol{u}^{g}, \delta \boldsymbol{u}^{f}$ and $\delta \boldsymbol{u}^{o}$ denote the virtual displacement on the particular boundary. 


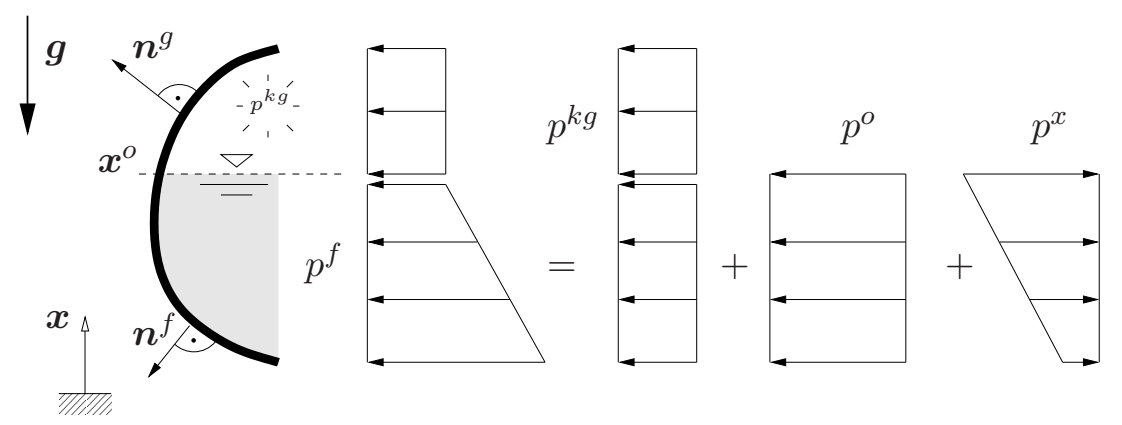

Bild 2.2: Hydrostatic pressure distribution in a chamber filled with gas and an incompressible heavy fluid

\subsubsection{Incompressible fluid with free fluid surface and additional gas loa- ding}

The gas and fluid pressure distribution for this case is depicted in figure 2.2. Using equations (2.7) and (2.8) in (2.2) gives us along with the pressure values

$$
\begin{aligned}
& p^{x}=\rho \boldsymbol{g} \cdot \boldsymbol{x} \\
& p^{o}=\rho \boldsymbol{g} \cdot \boldsymbol{x}^{o} \\
& p^{f}=p^{o}-p^{x}-p^{k g}
\end{aligned}
$$

the virtual gas and fluid potential for an incompressible fluid with free fluid surface and additional gas loading

$$
\delta \Pi^{f s i}=\int_{\eta^{f}} \int_{\xi^{f}} p^{f} \boldsymbol{n}^{f} \cdot \delta \boldsymbol{u}^{f} d \xi d \eta-\int_{\eta^{g}} \int_{\xi^{g}} p^{k g} \boldsymbol{n}^{g} \cdot \delta \boldsymbol{u}^{g} d \xi d \eta .
$$

\section{Linearization}

The virtual expression (2.12) is now written as a Taylor series expansion up to the first order term in order to embed it into a numerical solution procedure, e.g. in a Newton-type scheme. A consequent linearization of the virtual work (2.12) at a given state $t$ then leads to

$$
\begin{aligned}
\delta \Pi_{\text {lin }}^{f s i}= & \int_{\eta^{f}} \int_{\xi^{f}} p_{t}^{f} \boldsymbol{n}_{t}^{f} \cdot \delta \boldsymbol{u}^{f} d \xi d \eta+\int_{\eta^{g}} \int_{\xi^{g}} p_{t}^{k g} \boldsymbol{n}_{t}^{g} \cdot \delta \boldsymbol{u}^{g} d \xi d \eta \\
& \bullet \text { Pressure change parts } \\
+ & \int_{\eta^{f}} \int_{\xi^{f}} \Delta p^{f} \boldsymbol{n}_{t}^{f} \cdot \delta \boldsymbol{u}^{f} d \xi d \eta+\int_{\eta^{g}} \int_{\xi^{g}} \Delta p^{k g} \boldsymbol{n}_{t}^{g} \cdot \delta \boldsymbol{u}^{g} d \xi d \eta \\
& \bullet \text { Normal change parts } \\
+ & \int_{\eta^{f}} \int_{\xi^{f}} p_{t}^{f} \Delta \boldsymbol{n}^{f} \cdot \delta \boldsymbol{u}^{f} d \xi d \eta+\int_{\eta^{g}} \int_{\xi^{g}} p_{t}^{k g} \Delta \boldsymbol{n}^{g} \cdot \delta \boldsymbol{u}^{g} d \xi d \eta
\end{aligned}
$$




\subsection{Incremental pressure changes}

The incremental pressure changes can also be directly adopted from [13], [7]. The mass conservation of the fluid and the adiabatic state equation of an ideal gas with the isentropic exponent $\kappa$ then lead to the change of the gas pressure

$$
\Delta p^{k g}=-\alpha_{t} \int_{\eta^{g}} \int_{\xi^{g}} \boldsymbol{n}_{t}^{g} \cdot \Delta \boldsymbol{u}^{g} d \xi d \eta-\alpha_{t} \int_{\eta^{f}} \int_{\xi^{f}} \boldsymbol{n}_{t}^{f} \cdot \Delta \boldsymbol{u}^{f} d \xi d \eta,
$$

with

$$
\alpha_{t}=\kappa \frac{p^{k g}}{\bar{v}^{g}},
$$

the change of the pressure at the fluid surface

$$
\Delta p^{o}=-\gamma_{t} \int_{\eta^{f}} \int_{\xi^{f}} \boldsymbol{n}_{t}^{f} \cdot \Delta \boldsymbol{u}^{f} d \xi d \eta,
$$

with

$$
\gamma_{t}=-\rho_{t}\left(\int_{\eta^{f}} \int_{\xi^{f}} \boldsymbol{n}_{t}^{f} \cdot \boldsymbol{g} d \xi d \eta\right)^{-1}
$$

and the change of the position dependend pressure

$$
\Delta p^{x}=\rho_{t} \boldsymbol{g} \cdot \Delta \boldsymbol{u}^{f} .
$$

\subsection{Normal change parts}

The parts for the gas and fluid domains involving the change of the normals of the wetted surface parts can be adopted from [11] and [12]. For the fluid domain the specific part due to the non-constant pressure distribution $p_{t}^{x}$ must be considered [15]. By introducing the skewsymmetric tensors (see also [14] and [9])

$$
\begin{aligned}
\boldsymbol{W}^{\bar{\xi}} & =\boldsymbol{n}_{t} \otimes \boldsymbol{x}^{\bar{\xi}}-\boldsymbol{x}^{\bar{\xi}} \otimes \boldsymbol{n}_{t} \\
\boldsymbol{W}^{\bar{\eta}} & =\boldsymbol{n}_{t} \otimes \boldsymbol{x}^{, \bar{\eta}}-\boldsymbol{x}^{\bar{\eta}} \otimes \boldsymbol{n}_{t}
\end{aligned}
$$

in a convective basis $\boldsymbol{x}^{, \bar{\xi}} \otimes \boldsymbol{x}^{, \bar{\eta}}$, with $\bar{\xi}, \bar{\eta}$ as the contravariant curvilinear coordinates, we obtain the normal change parts of the linearized virtual work as follows:

$$
\begin{aligned}
\delta \Pi_{l i n}^{\Delta n^{g}}= & \int_{\eta^{g}} \int_{\xi^{g}} \frac{p_{t}^{k g}}{2}\left(\begin{array}{c}
\delta \boldsymbol{u}^{g} \\
\delta \boldsymbol{u}_{, \xi}^{g} \\
\delta \boldsymbol{u}_{, \eta}^{g}
\end{array}\right) \cdot\left(\begin{array}{ccc}
\mathbf{0} & \boldsymbol{W}^{\bar{\xi}} & \boldsymbol{W}^{\bar{\eta}} \\
\boldsymbol{W}^{\bar{\xi} T} & \mathbf{0} & \mathbf{0} \\
\boldsymbol{W}^{\bar{\eta} T} & \mathbf{0} & \mathbf{0}
\end{array}\right)\left(\begin{array}{c}
\Delta \boldsymbol{u}^{g} \\
\Delta \boldsymbol{u}_{, \xi}^{g} \\
\Delta \boldsymbol{u}_{, \eta}^{g}
\end{array}\right) d \xi d \eta \\
\delta \Pi_{l i n}^{\Delta n^{f}}= & \int_{\eta^{f}} \int_{\xi^{f}} \frac{p_{t}^{f}}{2}\left(\begin{array}{c}
\delta \boldsymbol{u}^{f} \\
\delta \boldsymbol{u}_{, \xi}^{f} \\
\delta \boldsymbol{u}_{, \eta}^{f}
\end{array}\right) \cdot\left(\begin{array}{ccc}
\mathbf{0} & \boldsymbol{W}^{\bar{\xi}} & \boldsymbol{W}^{\bar{\eta}} \\
\boldsymbol{W}^{\bar{\xi} T} & \mathbf{0} & \mathbf{0} \\
\boldsymbol{W}^{\bar{\eta} T} & \mathbf{0} & \mathbf{0}
\end{array}\right)\left(\begin{array}{c}
\Delta \boldsymbol{u}^{f} \\
\Delta \boldsymbol{u}_{, \xi}^{f} \\
\Delta \boldsymbol{u}_{, \eta}^{f}
\end{array}\right) d \xi d \eta \\
& -\int_{\eta^{f}} \int_{\xi^{f}}\left[p_{t, \xi}^{f}\left(\boldsymbol{x}_{, \eta} \times \delta \boldsymbol{u}_{, \xi}^{f}\right)-p_{t, \eta}^{f}\left(\boldsymbol{x}_{, \xi} \times \delta \boldsymbol{u}_{, \eta}^{f}\right)\right] \cdot \Delta \boldsymbol{u}^{f} d \xi d \eta
\end{aligned}
$$




\subsection{Proof of Conservativeness}

As shown in [14] and [9] by partial integration and subsequent application of the Gauss theorem the formally unsymmetric part in equation (3.10) can be transformed into a skew symmetric field term and an unsymmetric boundary term. Assuming physically realistic boundary conditions makes this boundary term to vanish. A complete symmetry of the linearized virtual work $\delta \Pi_{\text {lin }}^{f s i}$ is finally obtained, if the linearized pressure parts (3.2), (3.4) and (3.6) along with the normal change parts (3.9) and (3.10) are inserted in the initial equation (3.1). Further on, if $\rho_{t} \boldsymbol{g} \cdot \boldsymbol{\Delta} \boldsymbol{u}$ from equation (3.6) is split up into a symmetric and a skewsymmetric part, this skewsymmetric part neutralizes with the remainig skewsymmetric term in (3.10). Thus only the symmetric part from the position dependent pressure over the fluid boundary remains. The result clearly proves formally the conservativeness of the complete problem, which by general physical consideration is conservative from the origin.

The symmetry of the derived equations will appear clearer after the following FEdiscretization.

\subsection{Finite Element Mapping}

After discretizing the linearized virtual fluid structure interaction expression with isoparametric mapping functions $\boldsymbol{N}$ for the displacements, the virtual displacements and the incremental displacements of the structural parts which are in contact with gas/fluid, such that

$$
\boldsymbol{u}=\boldsymbol{N} \boldsymbol{d}, \quad \delta \boldsymbol{u}=\boldsymbol{N} \delta \boldsymbol{d} \quad \text { and } \quad \Delta \boldsymbol{u}=\boldsymbol{N} \Delta \boldsymbol{d},
$$

we obtain from the normal change parts the symmetric load stiffness matrices for each structural element in contact with gas and/or fluid (see also [15], [9], [10], [11], $[12])$ :

$$
\begin{aligned}
\boldsymbol{K}_{\text {elem }}^{g}= & \frac{1}{2} \int_{\eta^{g}} \int_{\xi^{g}} p_{t}^{g}\left(\begin{array}{c}
\boldsymbol{N} \\
\boldsymbol{N}_{, \xi} \\
\boldsymbol{N}_{, \eta}
\end{array}\right)^{T}\left(\begin{array}{ccc}
\mathbf{0} & \boldsymbol{W}^{\bar{\xi}} & \boldsymbol{W}^{\bar{\eta}} \\
\boldsymbol{W}^{\xi \bar{T}} & \mathbf{0} & \mathbf{0} \\
\boldsymbol{W}^{\eta \bar{T}} & \mathbf{0} & \mathbf{0}
\end{array}\right)\left(\begin{array}{c}
\boldsymbol{N} \\
\boldsymbol{N}_{, \xi} \\
\boldsymbol{N}_{, \eta}
\end{array}\right) d \xi d \eta(3,12 \\
\boldsymbol{K}_{\text {elem }}^{f}= & \frac{1}{2} \int_{\eta^{f}} \int_{\xi^{f}} p_{t}^{f}\left(\begin{array}{c}
\boldsymbol{N} \\
\boldsymbol{N}_{, \xi} \\
\boldsymbol{N}_{, \eta}
\end{array}\right)^{T}\left(\begin{array}{ccc}
\mathbf{0} & \boldsymbol{W}^{\bar{\xi}} & \boldsymbol{W}^{\bar{\eta}} \\
\boldsymbol{W}^{\xi \bar{T}} & \mathbf{0} & \mathbf{0} \\
\boldsymbol{W}^{\eta \bar{T}} & \mathbf{0} & \mathbf{0}
\end{array}\right)\left(\begin{array}{c}
\boldsymbol{N} \\
\boldsymbol{N}_{, \xi} \\
\boldsymbol{N}_{, \eta}
\end{array}\right) d \xi d \eta \\
& -\frac{\rho_{t}}{2} \int_{\eta^{f}} \int_{\xi^{f}} \boldsymbol{N}^{T}\left(\boldsymbol{n}_{t}^{f} \otimes \boldsymbol{g}+\boldsymbol{g} \otimes \boldsymbol{n}_{t}^{f}\right) \boldsymbol{N} d \xi d \eta
\end{aligned}
$$

The residual for the corresponding element results in negative right hand side vectors due to fluid and gas pressure loading/support following the definitions of the normals given in figure 2.1:

$$
\begin{aligned}
& \boldsymbol{f}_{\text {elem }}^{g}=-\int_{\eta^{g}} \int_{\xi^{g}} p_{t}^{k g} \boldsymbol{N}^{T} \boldsymbol{n}_{t}^{g} d \xi d \eta, \\
& \boldsymbol{f}_{\text {elem }}^{f}=\int_{\eta^{f}} \int_{\xi^{f}}\left(-p_{t}^{k g}+p_{t}^{o}-p_{t}^{x}\right) \boldsymbol{N}^{T} \boldsymbol{n}_{t}^{f} d \xi d \eta .
\end{aligned}
$$


Hence along with the coupling vectors reflecting the closed volumes

$$
\begin{aligned}
& \boldsymbol{a}_{\text {elem }}=\int_{\eta^{g}} \int_{\xi^{g}} \boldsymbol{N}^{T} \boldsymbol{n}_{t}^{g} d \xi d \eta \quad \text { and } \\
& \boldsymbol{b}_{\text {elem }}=\int_{\eta^{f}} \int_{\xi^{f}} \boldsymbol{N}^{T} \boldsymbol{n}_{t}^{f} d \xi d \eta
\end{aligned}
$$

for the elements in contact with gas/fluid and after assembling all local arrays in their corresponding global arrays the linearized state of equilibrium for a closed single chamber system partially filled with an incompressible fluid and gas can be written as:

$$
\begin{aligned}
&\left(\boldsymbol{K}^{e l}+\boldsymbol{K}^{g}+\boldsymbol{K}^{f}+\alpha_{t}(\boldsymbol{a}+\boldsymbol{b})(\boldsymbol{a}+\boldsymbol{b})^{T}-\gamma_{t} \boldsymbol{b} \boldsymbol{b}^{T}\right) \Delta \boldsymbol{d}= \\
& \boldsymbol{f}^{e x}-\boldsymbol{f}^{e l}-\boldsymbol{f}^{f}-\boldsymbol{f}^{g}
\end{aligned}
$$

In equation (3.18) the volume dependence of the enclosed gas and fluid is reflected in the rank updates of the stiffness matrix with two dyadic products of the coupling vectors for each closed volume.

\subsection{Multichamber Problems}

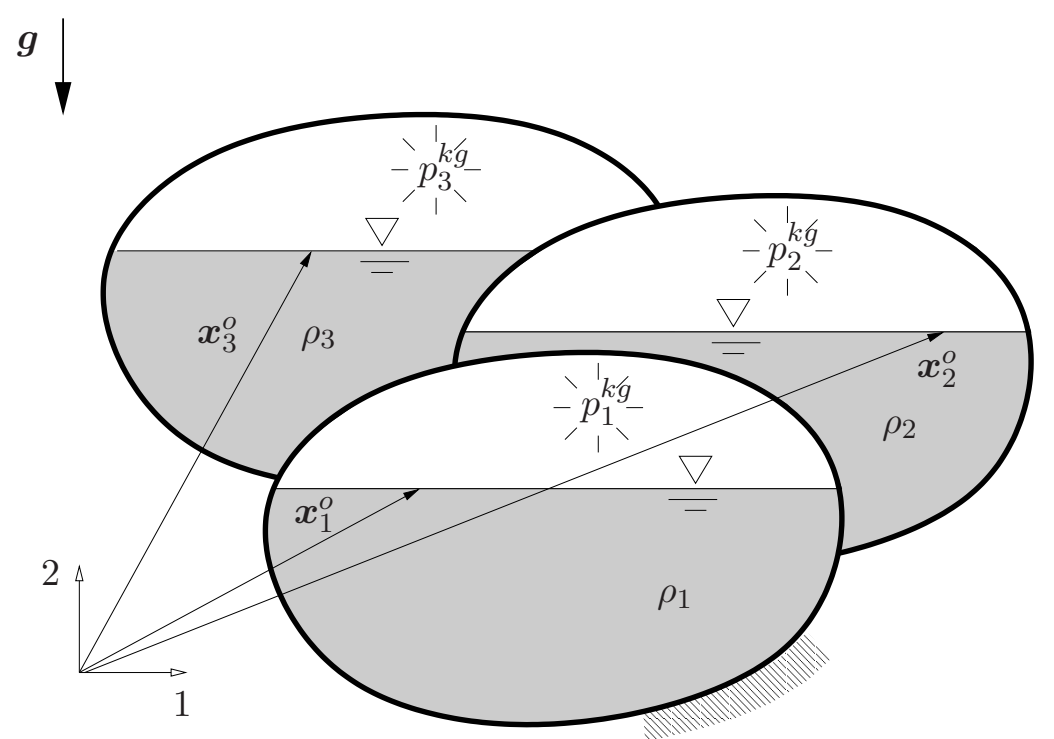

Bild 3.1: Multichamber problem with $n=3$ interacting fluid and gas filled chambers

The derived procedure can easily be expanded to problems, where multiple fluid and/or gas filled chambers are connected to each other. For such systems the corresponding global arrays (load-stiffness matrices, coupling vectors and right hand side vectors) have to be set up for each chamber and must be summed up for all $n$ chambers.

$$
\begin{array}{r}
\left(\boldsymbol{K}^{e l}+\sum_{i=1}^{n}\left[\boldsymbol{K}_{i}^{g}+\boldsymbol{K}_{i}^{f}+\alpha_{i, t}\left(\boldsymbol{a}_{i}+\boldsymbol{b}_{i}\right)\right.\right. \\
\left.\left.\left(\boldsymbol{a}_{i}+\boldsymbol{b}_{i}\right)^{T}-\gamma_{i, t} \boldsymbol{b}_{i} \boldsymbol{b}_{i}^{T}\right]\right) \Delta \boldsymbol{d} \\
=\boldsymbol{f}^{e x}-\boldsymbol{f}^{e l}-\sum_{i=1}^{n}\left[\boldsymbol{f}_{i}^{f}+\boldsymbol{f}_{i}^{g}\right]
\end{array}
$$


These multiple rank updates due to the volume dependence of the inner state variables of the fluid and gas lead to a fully populated system matrix. However, in order to save memory and computational effort during the solution process it is desirable to have matrices with band structure. Therefore in the next section a solution algorithm is presented, which benefits from the dyadic rank updates preserving the band structure.

\section{Solution}

In figure 4.1 a solution algorithm to solve equation systems like (3.19) with $n$ dyadic updates is briefly presented. A more detailled derivation of this procedure, which bases on a sequential application of the Sherman-Morrison formula, can be found in [7]. Most important in this recursive scheme, starting from the conventional solution $\Delta \boldsymbol{d}_{0}$, is that only a few vectors and scalars need to be stored within a single solution step, which keeps the memory requirements low.

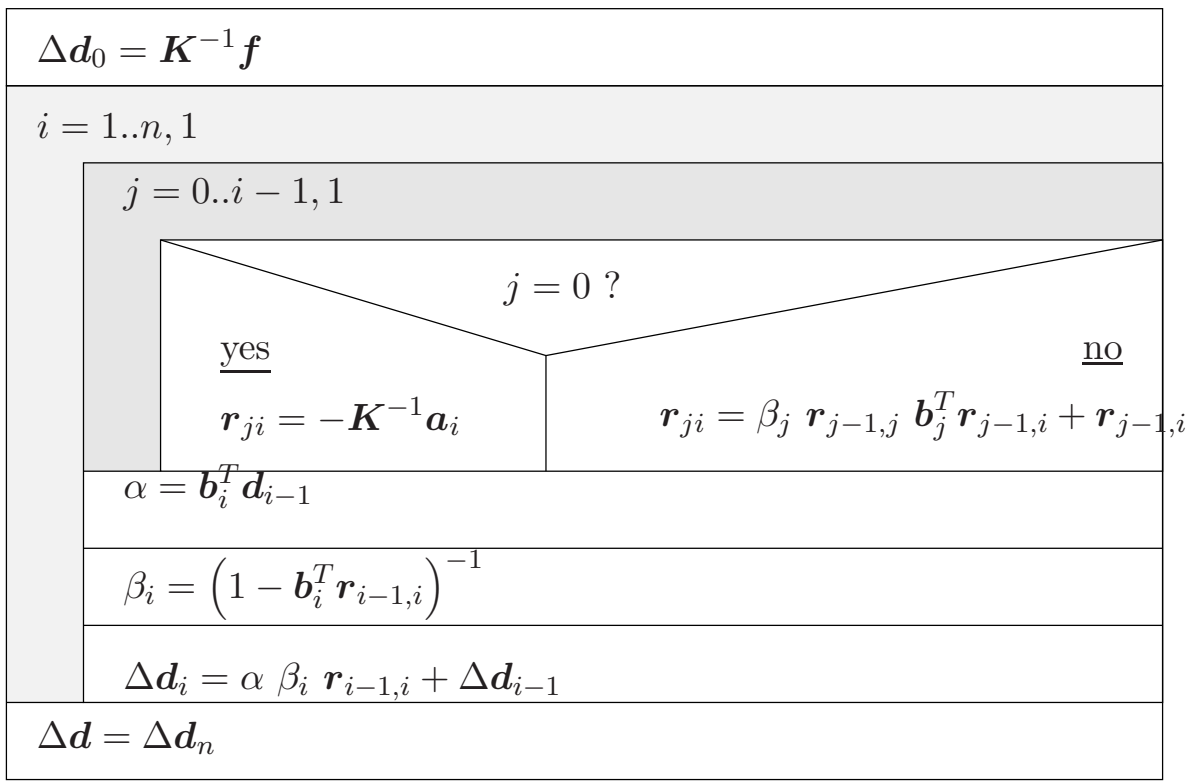

Bild 4.1: Recursive scheme for the computation of the auxiliary vectors $\boldsymbol{r}_{j i}$, the parameters $\beta_{i}$ and the interim solutions $\Delta \boldsymbol{d}_{i}$ in the vectorized Sherman-Morrison algorithm

\section{$5 \quad$ Numerical Examples}

The inflation and hydrostatic loading of a rubber dam under serveral boundary conditions will serve as an example with varying support and loading conditions. Figure 5.1 illustrates such an inflatable dam under hydrostatic loading: hydrodynamic effects will not be considered in these examples. The rubber dam consists of two membrane parts, which are hot volcanized along one edge resulting in the typical deflection fin, which is used to reduce vibrations. Along the other edge the two membrane layers are clamped and anchored to the ground. The most important task of weirs is the adjustment of the water retention level resp. the dam height, 
which allows to take control of the discharge. In the case of inflatable dams the dam height $h$ can be regulated by the internal gas pressure. Analytical solutions for different load cases can be found in [1] assuming purely membrane conditions and bending free joining of the membrane parts. The following computations to simula-

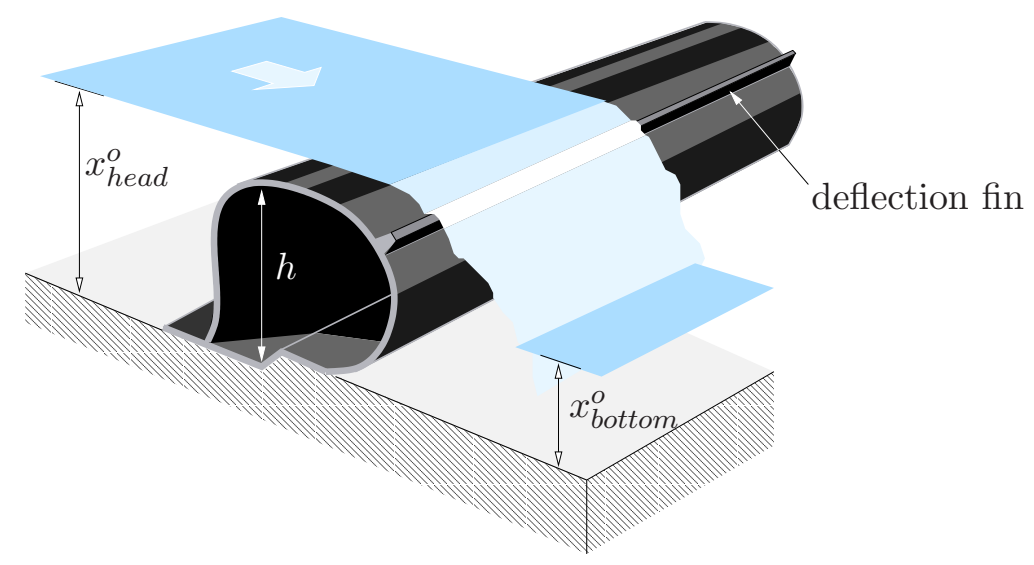

Bild 5.1: Sketch of inflated rubber dam under head water loading

te the development of the dam height during the loading process are performed in several steps: First the membrane structure is deployed by increasing the internal pressure. Afterwards the head and bottom water levels will be raised. The membranes for the dam (scale 1:10) have a thickness of $2 \mathrm{~mm}$ and a total circumference of about $850 \mathrm{~mm}$. The material data with Young's modulus $E=60 \mathrm{~N} / \mathrm{mm}^{2}$ and Poisson ration $\nu=0.4$ correspond to a fiber reinforced rubber membrane. In this special load case with head water loading, bottom water loading and an interior gas filling a 3-chamber system is the appropriate model. Although the head and bottom water levels are not affected by the deformation of the dam, which means that the rank updates of the system matrix result only from the gas filled chamber, the normal change parts and the residual vectors for all 3 chambers have to be considered. Further on boundary effects (e.g. wrinkling of membrane parts) in the axial direction of the dam are neglected in the analysis. Therefore we can restrict the investigation to a $2 \mathrm{D}$ model of the cross section discretized with 66 solid-shell elements [8] with linear shape functions and ANS/EAS enhancements for the kinematics. In future investigations the simulation of a fully 3D model of the dam will be performed, considering also the specific contact and support conditions at the boundaries leading often to wrinkling.

\subsection{Clamping along a single edge}

The first possible layout of an inflatable dam has a single clamped edge on the left (see figure 5.2). The body of the dam is in contact (penalty formulation) with the ground. The dam is pressurized until loading time"10, when the maximum dam height of $h=220 \mathrm{~mm}$ is reached (see figure 5.3) assuming static loading. Now the head water level is steadily increased, which in the end leads to some rolling motion 
of the dam, clearly visible in the decrease of the dam height of about $10 \%$. From loading time 20 on the downstream water level is slowly raised. As is visible in figure

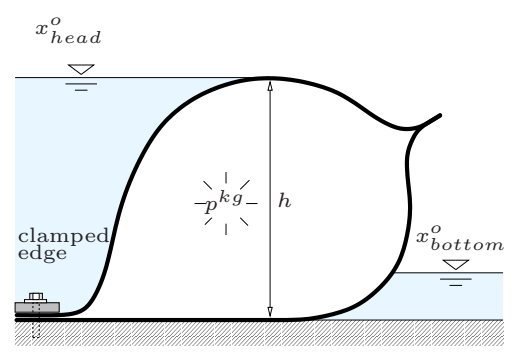

Bild 5.2: Cross section of rubber dam clamped along a single edge

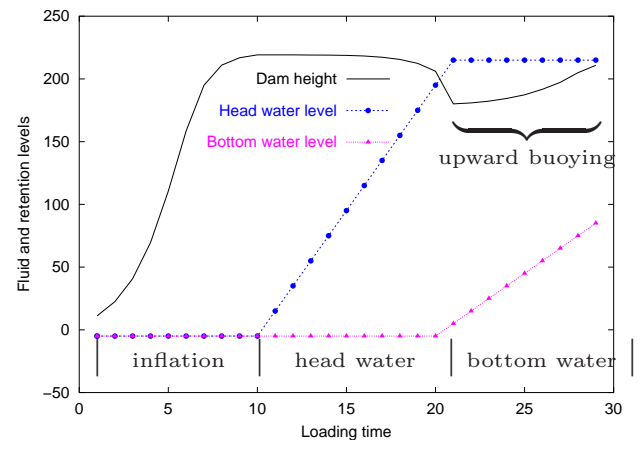

Bild 5.3: Development of dam height during loading process

5.3 the additional loading by the bottom water leads to an undesired buoyancy of the dam body, because the membrane is only clamped along the left edge. Two ways to overcome this buoyancy are presented in the next subsections.

\subsection{Clamping along a single edge and additional interior heavy fluid filling}

This example is similar to the previous one. But in order to avoid the upward buoying of the dam body its interior is filled with heavy fluid in addition to the gas filling (see figure 5.4). In figure 5.5 the development of the dam height during the loading process is given: In this case the interior fluid support reduces the dam height by a few percent. The last load increments of head water filling then again lead to a sudden decrease of about 10-15\% of the original height. But in contrast to the first example the plateau of the dam height curve shows that due to interior fluid support no further buoying effects appear.

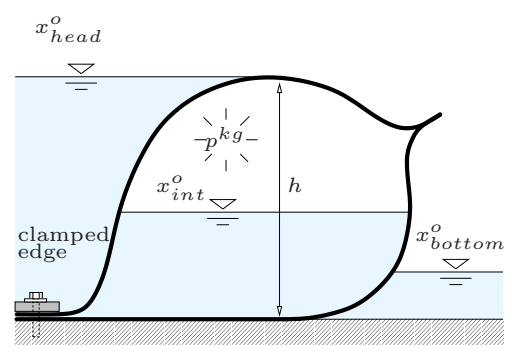

Bild 5.4: Cross section of rubber dam clamped along a single edge

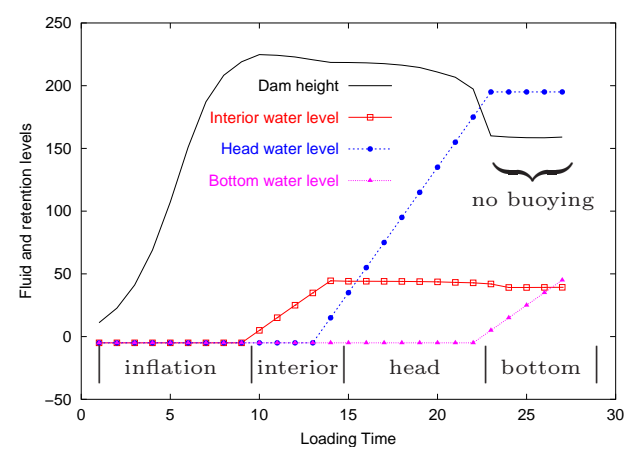

Bild 5.5: Development of dam height during loading and filling

\subsection{Clamping along two edges}

A second alternative to overcome the undesired upward buoying of the dam inflated by gas can be found by clamping another edge, as illustrated in figure 5.6. Looking 
again at the dam height development during the loading and filling process (figure 5.7) no visible effects of the loading on the retention level of the dam can be found. Although this may seem the most straight forward alternative to keep a constant dam height a disadvantage may be the rather large clamping forces. Therefore dams partially or fully filled with fluid may be preferable.

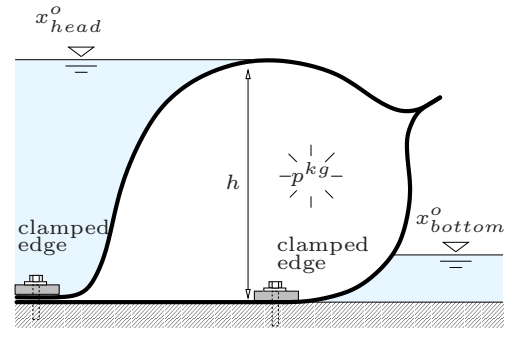

Bild 5.6: Cross section of rubber dam clamped along two edges

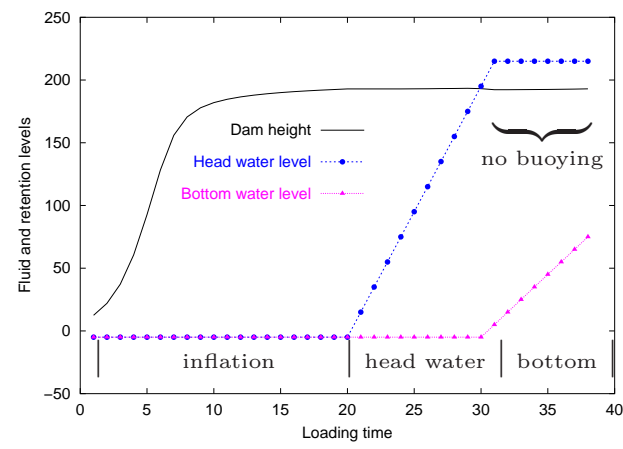

Bild 5.7: Development of dam height during loading process

\section{Conclusions}

In this contribution a meshfree description of a heavy incompressible fluid interacting with gas and a deformable enclosure was presented, which was based on a total energy functional. The formulation contains the change of inner state variables $p^{k g}$ and $\boldsymbol{x}^{o}$ over the deformation and therefore the model allows the simulation of large deformation processes for many kinds of fluid and gas loading as well as fluid/gas support, as shown in the numerical examples. The fluid-gas-structure-interaction was described via boundary integrals of the geometrical fluid and gas terms. Further both, the extension to multichamber problems and the combination of fluid and gas loading in single chambers was derived. An efficient solution algorithm for this kind of problems can be found in the Sherman-Morrison formula, which allows to avoid the inversion of the dyadic updated system matrix, which in addition may be badly conditioned in case of fluid support with a nearly incompressible fluid. The future work is to describe the possible separation of chambers during the deformation and the implementation of more complex contact algorithms to capture the more complex boundary conditions and folds at the side boundaries of the flexible dams. A further focus is on the stability investigation of gas/fluid loaded multichamber systems, especially inflatable dams, which often suffer from instability problems.

\section{Literatur}

[1] H. O. Anwar, Inflatable Dams. Journal of the Hydraulics Division, ASCE, 93, 99-119, 1967.

[2] J. Bonet, R. D. Wood, J. Mahaney, P. Heywood, Finite element analysis of air supported membrane structures. Computer Methods in Applied Mechanics and Engineering, 190, 579-595, 2000. 
[3] J. D. Faires and R. L. Burden, Numerische Methoden. Spektrum Akademischer Verlag, 1994.

[4] R. E. Freeland and G. D. Bilyeu and G. R. Veal and M. M. Mikulas, Inflatable deployable space structures Technology summary. International Aeronautical Federation, IAF-98-1.5.01, 1998.

[5] M. Gebhardt, Hydraulische und statische Bemessung von Schlauchwehren. PhDthesis, Institut für Wasserwirtschaft und Kulturtechnik der Universität Karlsruhe (TH), 2005 (in German).

[6] R.H. Luchsinger and A. Pedretti and A. Steingruber and M. Pedretti, The new structural concept Tensairity: Basic principles. Proceedings of the Second Conference of Structural Engineering, Mechanics and Computation, A.A. Balkema/Swets Zeitlinger, Lisse, 2004.

[7] M. Haßler and K. Schweizerhof, On the Interaction of Fluid and Gas Loaded Multi-Chamber Systems in Large Deformation Finite Element Analyses (accepted for publication). 2006.

[8] R. Hauptmann and K. Schweizerhof, A systematic development of 'solid-shell' element formulations for linear and non-linear analysis employing only displacement degrees of freedom. International Journal for Numerical Methods in Engineering, 42, 49-69, 1998.

[9] K. Knebel, Stabilität von Stahlzylindern mit unilateralen Randbedingungen bei statischen und dynamischen Beanspruchungen. PhD-thesis, Institut für Mechanik der Universität Karlsruhe (TH), 1997 (in German).

[10] T. Rumpel, Effiziente Diskretisierung von statischen Fluid-Struktur-Problemen bei großen Deformationen. PhD-thesis, Institut für Mechanik der Universität Karlsruhe (TH), 2003 (in German).

[11] T. Rumpel and K. Schweizerhof, Volume-dependent pressure loading and its influence on the stability of structures. International Journal for Numerical Methods in Engineering, 56, 211-238, 2003.

[12] T. Rumpel and K. Schweizerhof, Hydrostatic fluid loading in non-linear finite element analysis. International Journal for Numerical Methods in Engineering, 59, 849-870, 2004.

[13] T. Rumpel, K. Schweizerhof and M. Haßler, Efficient Finite Element Modelling and Simulation of Gas and Fluid Supported Membrane and Shell Structures. Textile Composites and Inflatable Structures, E. Onate, B. Kröplin (eds.), Springer, 2005.

[14] K. Schweizerhof, Nichtlineare Berechnung von Tragwerken unter verformungsabhängiger Belastung. PhD-thesis, Institut für Baustatik der Universität Stuttgart, 1982 (in German).

[15] K. Schweizerhof and E. Ramm, Displacemant dependent pressure loads in nonlinear finite element analyses. Computers \& Structures, 18, 1099-1114, 1984. 
[16] K. Schweizerhof, Quasi-Newton Verfahren und Kurvenverfolgungsalgorithmen für die Lösung nichtlinearer Gleichungssysteme in der Strukturmechanik. Institut für Baustatik der Universität Karlsruhe (TH), 1989 (in German).

[17] P. Wriggers, Nichtlineare Finite-Element-Methoden. Springer-Verlag Berlin, 2001.

[18] H. Ziegler, An introduction to thermomechanics. North-Holland Publishing Company, 1977.

[19] O. C. Zienkiewicz and R. L. Taylor, The finite element method, Vol. 1. Butterwoth-Heinemann, 2000. 